\title{
La metodología de los sistemas complejos de Rolando García aplicada a la investigación en actividad física y salud
}

\section{Martin Farinola ${ }^{1}$}

Universidad Nacional de La Matanza

\section{Tipo de trabajo: Artículo}

Material original autorizado para su primera publicación en Journal de Ciencias Sociales, Revista Académica de la Facultad de Ciencias Sociales de la Universidad de Palermo.

Recibido: 25-5-2014

Aceptado: 26-8-2014

\section{Resumen}

La insuficiente actividad física de la población es un problema de salud pública creciente. Su solución excede al campo de la salud llegándose a sugerir un enfoque interdisciplinario para su investigación. En este trabajo nos proponemos estudiar si es posible investigar la conducta en actividad física con fines de salud aplicando la metodología de los sistemas complejos propuesta por Rolando García.

Se hará un repaso histórico del proceso de investigación en actividad física y salud y a continuación se discutirá la pertinencia de un abordaje sistémico siguiendo la propuesta de Rolando García.

Llegamos a la conclusión de que la actividad física para la salud es un objeto abordable mediante la metodología propuesta por García. Esto es así debido a que reúne las características necesarias para una investigación sistémica e interdisciplinaria, estas son: los componentes y niveles son identificables; el sistema cuenta con una historia de transformaciones y reestructuraciones; existe investigación disciplinaria y especialistas en cada área involucrada; hay urgencia por parte de agentes gubernamentales y distintas instituciones por resolver el problema.

El desafío será entonces construir un equipo de trabajo preparado y con objetivos y marcos epistemológicos similares. La universidad podría ser un espacio propicio para formar un equipo de investigación interdisciplinario de estas características.

Palabras clave: Actividad física, salud, interdisciplina, metodología

1 Docente-investigador. Universidad Nacional de La Matanza. E-mail :martinfarinola@ymail.com 


\section{Abstract}

Insufficient physical activity of the population is a growing public health problem. Its solution exceeds the health field getting itself to suggest an interdisciplinary approach to its investigation. In this work we study whether it is possible to investigate physical activity behavior for health purposes using the methodology of complex systems proposed by Rolando Garcia.

Will be made a historical overview of the research process in physical activity and health and then will be discussed the suitability of a systemic approach as the proposed by Rolando Garcia.

We conclude that physical activity for health is an approachable object using the methodology proposed by Garcia. This is because it meet the necessary characteristics for a systemic and interdisciplinary research, these are: components and levels are identifiable; the system has a history of changes and restructuring; there are disciplinary research and specialists in each involved area; there is urgency by government agents and other institutions to solve the problem.

The challenge will be to build a prepared task force with similar objectives and similar epistemological frameworks. The university could be a suitable space to form an interdisciplinary research team of this nature.

Keywords: Physical activity, health, interdiscipline, methodology.

\section{Introducción}

La actividad física ha sido definida recientemente como la conducta que involucra el movimiento corporal y que resulta en un incremento del gasto energético y eventualmente de la condición física (adaptado de Pettee Gabriel, Morrow y Woolsey, 2012).

Si bien la actividad física se ha estudiado en distintos campos del conocimiento, el campo de la salud ha sido el que más atención le ha prestado y el que más ha influido en acrecentar el interés por su estudio en otras disciplinas. En efecto la actividad física ha resultado ser eficaz en la prevención primaria y secundaria de numerosas enfermedades crónicas no transmisibles (ECNT) tales como las enfermedades cardiovasculares, diabetes, ciertos tipos de cáncer, hipertensión, obesidad, depresión y osteoporosis (DHHS, 2008; Warburton, Nicol \& Verdín, 2006).

En la Argentina, la actividad física insuficiente tiene una elevada prevalencia (Ferrante, Linetzky, Konfino, King, Virgolini \& Laspiur, 2011), la cual no existió siempre ni se conformó en un instante. La actividad física habitual ha ido disminuyendo en los últimos siglos, y especialmente en las últimas décadas, tanto local como globalmente. Existe preocupación por recuperar un estilo de vida en el cual se reincorpore una mínima cantidad de actividad física que permita prevenir o retrasar la aparición de las enfermedades mencionadas más arriba (OMS, 2010). Este problema no sólo no se ha solucionado sino que se encuentra creciendo, por lo que existe un llamado a estrategias de investigación novedosas que encuentren luz en esta cuestión.

En este trabajo nos proponemos estudiar hasta qué punto podría investigarse la conducta en actividad física aplicando la metodología de los sistemas complejos propuesta por Rolando García. 


\section{Argumentación teórica}

Investigación en actividad física y salud: síntesis histórica. El campo de la investigación en actividad física y salud se encuentra en continua evolución desde que Jeremy Morris publicó sus primeros trabajos epidemiológicos a mediados del siglo pasado (Morris, Heady, Raffle, Roberts \& Parks, 1953). Desde aquel entonces a la fecha, uno de los acontecimientos más significativos que ocurrió fue la publicación en 1995 del artículo titulado Actividad física y salud pública, Recomendación del Centro para el Control y Prevención de Enfermedades y del Colegio Americano de Medicina del Deporte (Pate, Pratt, Blair, et al., 1995). En dicha publicación se presentó evidencia sólida que indicaba que con sólo acumular 30 minutos por día de actividad física a intensidad moderada, continuos o fraccionados en bloques de 10 minutos, era suficiente como para obtener beneficios en salud. Hasta dicha publicación las recomendaciones de actividad física para población general sólo hacían referencia a la cantidad de ejercicio necesario para mejorar la aptitud física (ACSM, 1990) y, como esta cantidad implicaba una intensidad, duración y frecuencia semanal importantes, sólo aquellas personas que tuvieran el tiempo y la motivación suficientes llevarían a cabo la actividad física recomendada.

La importancia de la publicación de 1995 radica en que se descubrió que no hacen falta grandes cantidades de tiempo ni de esfuerzo para que la actividad física llegue a producir beneficios, y que por lo tanto casi cualquier persona en cualquier situación puede promover su salud por medio de la actividad física. Esto, junto con la creciente incidencia de enfermedades crónicas asociadas a la insuficiente actividad física, hizo aumentar el interés de distintas instituciones por la promoción de la actividad física (DHHS, 1996; NIH, 1996).

Rápidamente se advirtió que el conocimiento de la dosis mínima de actividad física para promover la salud no resulta suficiente (aunque sí necesario) para que sujetos insuficientemente activos aumenten su nivel de actividad. Además también se advirtió que el campo de la promoción de la actividad física es un campo de estudio emergente y, casi por definición, interdisciplinario.

Se necesitan intervenciones creativas para ayudar a individuos sedentarios a volverse más activos. Estamos convencidos que los científicos del ejercicio y los profesionales de la salud pública deben integrar más fuertemente los conocimientos y los métodos de las ciencias de la conducta a las intervenciones en actividad física. (Blair \& Morrow, 1998, p. 255).

A principios de este milenio Sallis, Linton y Kraft (2005) reconocen una nueva fase en el proceso de investigación en actividad física y salud. Esta nueva fase está enfocada al entendimiento y consecuente modificación de factores políticos y de entorno que han llevado a la conformación de este estilo de vida de baja actividad física en los países industrializados. La idea principal pasa por facilitar la obtención de un estilo de vida que lleve la actividad física a la vida cotidiana, superando el tradicional estudio del tiempo libre y comenzando a trabajar sobre los hábitos en otros dominios como por ejemplo el transporte o el hogar. Pero para esto se hizo inevitable interactuar con sectores con los que no se solía hacerlo. 
La ampliación del concepto de actividad física y el creciente énfasis puesto en el estudio de factores políticos y de contexto han requerido la creación de nuevas colaboraciones investigativas con disciplinas y sectores de la sociedad que nunca antes habían interactuado con investigadores de la actividad física (Sallis, et al., 2005, p. S93).

La aparición de estos nuevos problemas (más políticos y de gestión) y de este nuevo modo de investigar en actividad física pareció ser el resultado de una secuencia lógica. Primero supimos cuánta actividad física hacer, luego qué hacer para promoverla, y ahora necesitamos saber cómo poner en práctica los conocimientos científicos para mejorar la realidad social, que en este caso consistiría en facilitar la adquisición de un estilo de vida más activo y saludable.

Por lo que, en el caso del proceso de investigación en actividad física y salud, los métodos y modos de investigación utilizados han sido aquellos capaces de solucionar los problemas que se fueron planteando, es decir "los métodos fueron siguiendo a los problemas" (Farinola y Bazán, 2011).

En este punto es en donde creemos que para el abordaje de la problemática de la actividad física es necesario ensayar metodologías de investigación que permitan un abordaje interdisciplinario, en el sentido de promover la interacción de propiedades pertenecientes a diferentes disciplinas, tanto sociales como naturales. Lo que resta del artículo se dedicará a ensayar una justificación acerca de la pertinencia de aplicar una metodología para sistemas complejos a esta problemática: la metodología propuesta por Rolando García.

Características de la investigación contemporánea en actividad física y salud que la hacen requerir un abordaje sistémico.

En el breve repaso histórico anterior se dejan ver elementos que hacen factible que la metodología de los sistemas complejos se pueda aplicar a la investigación contemporánea en actividad física y salud. Algunas de esas características son:

- Es una parcela de la realidad identificada y aceptablemente definida (las conductas en actividad física y su rol en la prevención de ECNT) pero que se encuentra inmersa en una realidad más amplia (el estilo de vida de las personas y el sistema de salud en general).

- Existen subsistemas interdefinibles también identificables: el núcleo familiar, el ámbito laboral/ escolar, el marco político-legal, la tecnología disponible, entre otros.

- Existen disciplinas diversas que han acumulado importante evidencia disciplinar acerca del tema: la fisiología, la psicología, la epidemiología, la antropología, entre otras.

Además se produce algo que Rolando García señala y es que si tenemos un objeto (sistema complejo) y una metodología (interdisciplina), la relación entre ambos sigue el siguiente orden: primero se define un sistema complejo y después se decide aplicar la interdisciplina; y no a la inversa que consistiría en contar con la interdisciplina como estrategia y luego ver dónde se aplica. "Llamaremos entonces investigación interdisciplinaria al tipo de estudio que requiere un sistema complejo" (García, 2006, 
p. 88). Esto es lo que, como se vio, sucedió con la investigación en actividad física: "los métodos fueron siguiendo a los problemas", dijimos más arriba. Es decir, se ha visto que la conducta en actividad física se relaciona con multiplicidad de aspectos interdefinibles que "obligan" a estudiarla sistémicamente.

A su vez este sistema que define a las conductas en actividad física tiene su historia. Los bajos niveles de actividad física que comúnmente se observan hoy en la población general no existieron siempre, son el resultado de un proceso de transformación cultural que ha ocurrido en la mayoría de los países desarrollados y que se está consolidando en los países en vías de desarrollo (Turconi \& Cena, 2007). Según García "la comprensión cabal del funcionamiento de un sistema complejo requiere un análisis de la historia de los procesos que condujeron al tipo de organización (estructura) que presenta en un momento dado" (García, 2006, p. 81). La historia de este sistema de actividad física puede ser tan larga como la humanidad misma, la cual en sus orígenes realizaba una cantidad elevada (elevada desde la mirada actual) de actividad física para subsistir, principalmente para cazar y recolectar pero también para conseguir refugio (Farinola, 2006). De ese entorno cazadorrecolector al entorno urbano actual, y pasando por las revoluciones neolítica e industrial, los niveles de actividad física se han ido transformando hasta alcanzar en la actualidad los valores más bajos de la historia.

Por otro lado, la investigación en actividad física y salud responde a otra característica que señala García y que requiere de un abordaje sistémico: "La investigación de un sistema complejo responde generalmente a una situación crítica frente a la cual, gobiernos, organizaciones y agencias de ayuda, deben intervenir de manera urgente" (García, 2006, pp. 34-35). Este es el caso de la baja actividad física a nivel global pero también local. En Argentina, la enfermedad cardiovascular es la primera causa de muerte y según el Ministerio de Salud, entre 2005 y 2009, se observó un incremento significativo de dos de sus factores de riesgo más importantes: la baja actividad física y la alimentación menos saludable.

Los resultados de la Encuesta Nacional de Factores de Riesgo 2009 reafirman la relevancia de las ECNT y sus factores de riesgo para la salud poblacional en Argentina. Las acciones de prevención y control deberán intensificarse, requiriendo además un cambio de paradigma para lograr resultados favorables (Ferrante et al., 2011, p. 40).

Es decir, revertir la tendencia hacia una disminución de la actividad física es un tema urgente, al menos en la arena de la salud pública.

\section{El componente epistemológico para el estudio de los sistemas complejos.}

Antes de discutir asuntos metodológicos es necesario plantear la epistemología desde la cual esa metodología va a adquirir valor, una epistemología desde la cual se desprendan las decisiones y los enfoques metodológicos que se propondrán.

Dando por superadas e infundadas las epistemologías aprioristas y empiristas, García (2006) 
propone una epistemología constructivista, siendo la versión más acabada la Epistemología Genética de Piaget. Según el autor se trata de la única teoría constructivista del conocimiento de carácter científico, independizada de la filosofía especulativa y basada y validada empíricamente. En resumen, dentro de esta concepción el niño construye conocimiento mediante la acción e interacción con los objetos del mundo exterior, y estos mecanismos son comunes, mutatis mutandis, a la construcción del conocimiento científico.

Esta concepción tiene consecuencias relevantes para la metodología. Una consecuencia primordial es que no hay una lectura pura de la experiencia. García diferencia entre "observable" y "percibido". Un "observable" supone mucho más que un registro perceptivo, supone una teoría desde la cual lo que se percibe adquiere sentido y entidad.

Otra consecuencia es la relación con la "realidad" exterior. Según García (2006) "el constructivismo puede definirse como un realismo epistemológico" (p. 84), es decir que se acepta la existencia de un mundo exterior con el cual interactuamos pero la forma de acceder a él es a través de un conocimiento construido. Esto se consigue recortando la realidad y estructurando la parcela de esa realidad que quedó dentro del recorte (sin olvidar las conexiones con lo que quedó por fuera de ese recorte). Dos principios guían este procedimiento: una disposición de sus elementos por niveles de organización con dinámicas propias, pero interactuantes entre sí; y una evolución que no procede por desarrollos continuos sino por reorganizaciones sucesivas. "De estos dos principios surgen pautas específicas para el ordenamiento metodológico de la investigación" (García, 2006, p. 80).

\section{- La metodología de los sistemas complejos aplicada a la investigación en actividad} física y salud.

"Un sistema complejo es una representación de un recorte de la realidad, conceptualizado como una totalidad organizada, en la cual los elementos no son separables y, por tanto, no pueden ser estudiados aisladamente" (García, 2006, p. 21). Este recorte de la realidad es conceptual, vale decir, no está aislado del contexto del cual es recortado. Esto significa que no es posible estudiar un sistema así definido sin tomar en cuenta sus interacciones con lo que hemos dejado fuera del recorte, aunque esas interacciones puedan quedar en un segundo plano por conveniencia. Justamente "una parte fundamental del esfuerzo de investigación es la construcción (conceptualización) del sistema como recorte más o menos arbitrario de una realidad que no se presenta con límites ni definiciones precisas" (García, 2006, p. 98).

Para el caso de la investigación en actividad física y salud, cuyo objetivo es lograr que los niveles de actividad física habitual de la población alcancen valores mínimos saludables, el sistema estaría conformado por la conducta en actividad física y sus características, sus resultantes individuales y comunitarias ${ }^{2}$, los factores intrínsecos (biológicos y psicológicos) que la afectan, y los elementos de contexto (físico y cultural) que la facilitan o la obstaculizan. Son paradigmáticos los estudios en comunidades aborígenes que han sido urbanizadas, en los cuales se han afectado sus contextos

\footnotetext{
2 Una resultante individual podría ser el aumento del gasto energético del sujeto que lleve adelante la actividad física; una resultante comunitaria podría ser una disminución de la presión sobre el sistema de salud debido a los beneficios para la salud que conlleva la práctica regular de la actividad física.
} 
físicos (pasaje de entorno rural a urbano), culturales (cambio de hábitos en el tiempo libre o las actividades laborales), ha disminuido su actividad física habitual y esto ocurrió paralelamente con un aumento de la obesidad y la diabetes (Bennett, 1999; Rode \& Shephard, 1994). Es decir que las conductas en actividad física están asociadas, no solo a factores intrínsecos del sujeto, sino también al entorno tanto físico como cultural. Esto muestra que ninguna disciplina por sí sola que se dedique a estudiar uno de los componentes del sistema (por ejemplo la fisiología o la geografía), podrá lograr una comprensión del sistema que permita modificarlo. Más arriba hicimos una cita textual que ilustra este concepto, los epidemiólogos haciendo un llamado a las ciencias de la conducta debido a que ellos por sí mismos no están en condiciones de resolver los cada vez más bajos niveles de actividad física de la población. De aquí se sigue que se torne necesario un abordaje interdisciplinario.

Los factores que directa o indirectamente determinan el funcionamiento de un sistema complejo pueden ser distribuidos en niveles estructuralmente diferenciados, con sus dinámicas propias. Cada nivel tiene una articulación interna y a su vez existe también una interacción entre niveles. Al nivel inmediato del problema empírico que se busque resolver García lo llama "nivel de base", que en este caso sería el nivel y características de la actividad física realizada por los sujetos. Por el otro lado, al conjunto de interacciones que ejercen influencia sobre un nivel dado García lo designó como las "condiciones de contorno" de dicho nivel. Estas interacciones entre niveles tienen lugar por medio de distintos tipos de influencias, no siempre materiales, llamadas "flujos". En este caso, las influencias del entorno físico (presencia/ausencia de espacios verdes cercanos, veredas en buenas condiciones, centros deportivos al alcance, etc.), del entorno cultural (hábitos lúdicos y de recreación, actividades "aceptables" para cada género, entre otras), del contexto político/legal (obligación de educación física escolar, ley de tránsito que favorezca los viajes a pie y en bicicleta como medios de transporte, ley de deporte, etc.) son elementos de otros niveles que conforman el sistema complejo bajo estudio. Estos elementos de cada nivel deben seleccionarse en la medida que exista evidencia disciplinaria acerca de su asociación con la conducta en actividad física, de esta manera los hechos observados son consistentes con el sistema así conformado.

Por último mencionamos que "no es suficiente una descripción del sistema, los estudios históricos son una herramienta indispensable en el análisis sistémico. La relación entre función y estructura (o entre estados y procesos) es la clave para la comprensión de los fenómenos" (García, 2006, p. 98). Como se mencionó más arriba, la historia de un sistema de conductas en actividad física puede iniciarse en los comienzos de la humanidad. Si definimos a la actividad física como la conducta que involucra al movimiento corporal y que conlleva un gasto energético, esto lo hemos hecho desde que el género homo emergió hace unos 2 millones de años, e incluso antes. Sin embargo, para comprender el funcionamiento del sistema hoy día, sería suficiente con comenzar el análisis en homo sapiens, cuyo estilo de vida original fue cazador-recolector de una elevada actividad física obligatoria para poder subsistir (Farinola, 2006). Este nivel de actividad física elevado se vio afectado en un principio por un cambio en los patrones de subsistencia: la sedentarización asociada al surgimiento de la agricultura. Con el tiempo, la incorporación de tecnologías que permitieron reducir los esfuerzos físicos en el trabajo, el transporte y en la vida cotidiana en general (palancas, poleas, animales de tracción, máquina de vapor, electricidad, control remoto, etc.) fogonearon la reducción de las conductas en actividad física de las poblaciones. El estudio histórico del sistema 
de conductas en actividad física permitirá una comprensión mayor del mismo en la actualidad y posibilitará reconocer los elementos más significativos de dicho sistema.

\section{Conclusiones}

Las conductas en actividad física han ido disminuyendo a lo largo de los últimos siglos, esto ha coadyuvado al aumento de la incidencia y prevalencia de ECNT. Las últimas tendencias de investigación para la promoción de la actividad física y la reversión de este fenómeno involucran a la interdisciplina como estrategia de abordaje del problema (Sallis, Linton \& Kraft, 2005). Sin embargo, y hasta nuestro conocimiento, no se ha formulado hasta la fecha un programa de investigación del tipo interdisciplinario siguiendo una metodología de abordaje de sistemas complejos ${ }^{3}$. En este trabajo se analizó la aplicabilidad de la propuesta de Rolando García de investigación interdisciplinaria de sistemas complejos al estudio de la conducta en actividad física con objetivos de salud.

Concluimos que este campo de investigación científica es abordable mediante la metodología propuesta por García ya que reúne las características necesarias para una investigación sistémica e interdisciplinaria, estas son: los componentes y niveles son identificables; el sistema cuenta con una historia de transformaciones y reestructuraciones; existe investigación disciplinaria y especialistas en cada área involucrada; hay urgencia por parte de agentes gubernamentales y de distintas instituciones locales y globales por resolver el problema de la prevención de ECNT.

El desafío es entonces construir un equipo de trabajo preparado y con objetivos y marcos epistemológicos similares. Este equipo debería formularse preguntas tales como: ¿Cómo se llegó a un estilo de vida de baja actividad física? ¿Qué sucesos históricos afectaron el nivel de actividad física de las poblaciones? ¿Cuáles son los elementos relacionados con las conductas en actividad física en la actualidad? ¿Qué elementos podrían incorporarse en el sistema para conducirlo hacia un estado de salud favorable de la población? A su vez el proceso de investigación mismo irá mejorando los conceptos, incorporando factores omitidos y eliminando elementos que aparecen como innecesarios o secundarios. Es decir que el sistema se irá precisando a medida que se avance en dar respuesta a estos interrogantes.

Una universidad podría ser el espacio propicio para formar un equipo de investigación interdisciplinario de estas características, ya que cuenta con facultades o departamentos disciplinares y con la misión de construir un conocimiento relevante socialmente.

\footnotetext{
${ }^{3}$ Abordajes similares son propuestos en los llamados modelos ecológicos de las conductas en salud, por ejemplo en Sallis, Owen \& Fisher (2008). Sería interesante hacer una comparación pormenorizada de la propuesta de García con la de estos autores con el fin de evaluar si efectivamente se trata de enfoques diferentes y qué tanto pueden complementarse.
} 


\section{Referencias bibliográficas}

ACSM (American College of Sports Medicine). (1990). Position Stand: The recommended quantity and quality of exercise for developing and maintaining cardiorespiratory and muscular fitness in healthy adults. Med Sci Sports Exerc, 22, 265-274.

Bennett, P. (1999). Type 2 diabetes among the Pima Indians of Arizona: An epidemic attributable to environmental change? Nutrition Reviews, 57, S51-54.

Blair, S. \& Morrow, J. (1998). Introduction to the Cooper Institute / The American College of Sports Medicine 1997 PhysicalActivity Interventions Conference. Am J Prev Med, 15, 255-256.

DHHS (Department of Health and Human Services). (1996). Centers for Disease Control and Prevention. National Center for Chronic Disease Prevention and Health Promotion. Physical Activity and Health: A Report of the Surgeon General. Atlanta, Estados Unidos. Consultado el 13/03/2014 de http://www.cdc.gov/nccdphp/sgr/pdf/sgrfull.pdf

DHHS (Department of Health and Human Services). (2008). Physical Activity Guidelines Advisory Committee. Physical Activity Guidelines Advisory Committee Report, 2008. Washington, DC, Estados Unidos. Consultado el 13/03/2014 de http://www.health.gov/paguidelines/Report/pdf/ CommitteeReport.pdf

Farinola, M. (2006). Explicación de un modelo integrador sobre la relación de causalidad entre la actividad física, la salud y el riesgo de muerte prematura. apunts Educación Física y Deportes, 85, 15-27. Consultado el 13/03/2014 de http://www.revista-apunts.com/es/hemeroteca?article=113

Farinola, M. y Bazán, N. (2011). El proceso contemporáneo de investigación en actividad física y salud: de la epidemiología a la interdisciplina. Perspectivas Metodológicas, Año 11, $\mathrm{n}^{\circ}$ 11, 97108.

Ferrante, D., Linetzky, B., Konfino, J., King, A., Virgolini, M., Laspiur, S. (2011). Encuesta Nacional de Factores de Riesgo 2009: evolución de la epidemia de enfermedades crónicas no transmisibles en Argentina. Rev Argent Salud Pública, 2, 34-41. Consultado el 13/03/2014 de http://www. saludinvestiga.org.ar/rasp/articulos/volumen6/encuesta-nacional.pdf

García, R. (2006). Sistemas complejos: Conceptos, método y fundamentación epistemológica de la investigación interdisciplinaria. Barcelona: Gedisa.

Morris, J., Heady, J., Raffle, P., Roberts, C., Parks, J. (1953). Coronary heart disease and physical activity of work. Lancet, ii, 1053-1057 and 1111-1120.

NIH (National Institutes of Health). (1996). Consensus Development Panel on Physical Activity and Cardiovascular Health. NIH Consensus Conference: Physical activity and cardiovascular health. JAMA, 276, 241-246.

OMS (Organización Mundial de la Salud). (2010). Recomendaciones mundiales sobre actividad física para la salud. Ginebra, Suiza. Consultado el 13/03/2014 de http://whqlibdoc.who.int/ publications/2010/9789243599977 spa.pdf?ua=1

Pate, R., Pratt, M., Blair, S., Haskell, W., Macera, C., Bouchard, C., et al. (1995). Physical activity and 
public health. JAMA, 273, 402-407.

Pettee Gabriel, K., Morrow, J. \& Woolsey, A. (2012). Framework for Physical activity as a complex and multidimensional behavior. Journal of Physical Activity and Health, 9(Suppl 1), S11-S18.

Rode, A. \& Shephard, R. (1994). Physiological consequences of acculturation: a 20-year study of fitness in an Inuit community. Eur J Appl Physiol, 69, 516-524.

Sallis, J., Linton, L. \& Kraft, K. (2005). The First Active Living Research Conference: Growth of a Transdisciplinary Field. Am J Prev Med, 28, S93-S95.

Sallis, J., Owen, N. \& Fisher, E. (2008). Ecological models of health behavior. En Glanz, Rimer, Viswanath (editors). Health behavior and health education. 4th Ed. Jossey-Bass: San Francisco, CA.

Turconi, G., Cena, H. (2007). Epidemiology of obesity. En Debasis Bagchi, Harry G. Preuss (eds). Obesity: Epidemiology, pathophysiology, and prevention. Boca Raton, FL, CRC PRESS.

Warburton, D., Nicol, C., Bredin, S. (2006). Health benefits of physical activity: The evidence. Can MedAsoc J, 174, 801-809. Consultado el 13/03/2014 de http://www.cmaj.ca/content/174/6/801. full.pdf+html 